Erschienen in: Stickel, Gerhard (Hrsg.): Deutsche Gegenwartssprache. Tendenzen und Perspektiven. - Berlin, New York: de Gruyter, 1990. S. 292-301. (Institut für deutsche Sprache. Jahrbuch 1989)

\title{
ALOIS BRANDSTETTER
}

\section{Über Qualitätsverlust und -konstanz in der Sprache, (dargestellt an Veränderungen im Wortfeld „Winter")}

"Kleinkirchheim schneit, Simonhöhe schneit noch nix", hörte ich in den Tagen vor Weihnachten 1988 jemanden im Cafehaus sagen. Ich verstand. Gemeint war nicht: „In Bad Kleinkirchheim schneit es”, sondern: Kleinkirchheim (Subjekt) schneit (Prädikat). Oder anders ausgedrückt: Der Wintersportort beschneit seine Pisten, bläst ein weißes Band künstlichen Schnees aus Schneekanonen in die braungrüne Landschaft. Von diesem neuen Gebrauch des alten Wortes schneien wissen meine Grammatiken wieder einmal nichts. Sie machen sich im Rahmen der sogenannten "Impersonalia", das heißt der "unpersönlichen Zeitwörter", im besonderen im Zusammenhang der "Witterungsimpersonalia" Gedanken darüber, wer sich eigentlich hinter dem unpersönlichen es versteckt. (Es regnet, es schneit, es donnert, es dämmert etc.), und ob man berechtigt sei, überhaupt von einem „Subjekt" zu sprechen:

Während manche Forscher dem "es" gar keinen inhaltlichen Wert zuerkennen und es als reines Formwort oder Scheinsubjekt bezeichnen, erblicken andere in ihm den sprachlichen Ausdruck für das Wirken unpersönlicher, irrationaler oder mythischer Kräfte

(Duden Die Grammatik 1973).

Früher ließ Petrus schneien, könnte man sagen, heute schneit Bad Kleinkirchheim selbst.

Wer schneien will, braucht also eine „Schneekanone”. Ohne Kanone kann der faulen Natur der Krieg nicht angesagt werden. So kriegen wir, was wir nicht bekommen. Leise rieselt der Schnee? Mächtig donnert die Kanone. Sanfter Tourismus schau oba. Den Winter gibt es nur noch in der romantischen Poesie, im modernen Geschäftsleben ist er die Skisaison mit Schneegarantie: Ski und Rodel gut. Der Schneebericht ist der eigentliche Bericht zur Lage der Skination.

Vor einigen Jahren war ich zur Winterszeit, respektive Skisaison, einmal in Innsbruck, um in einer Buchhandlung aus meinem Buch "Vom Schnee der vergangenen Jahre" vorzulesen. Vor meinem literarischen Auftritt vertrat ich mir noch ein wenig die Beine. Als ich zufälig am Kongreßhaus vorbeikam, sah ich dort eine Ausstellung von Pistenraupen, Schneekanonen, Fräsen und anderen sinnreichen Maschinen, deren Funktion ich gar nicht kannte. Zwischen den Apparaten zum Heranschaffen und zum Fortschaffen des Schnees aber standen Bürgermeister 
und Fremdenverkehrsdirektoren und andere Schneesachverständige und bestellten bei den Firmenvertretern die neueste Generation von Schneegeneratoren. Von "Helicopter-Skiing" sprachen die kernigen Tiroler, von "Snowboard", "Lifting", alles in Tyrolean English. Und schon damals stellten sich mir zwei Fragen: Wie lange hält die Natur diesen Wintersport aus? Die zweite Frage aber hat Günther Drosdowski, der Leiter der Dudenredaktion zum Thema eines Vortrages gemacht, den mir die F.A. Brockhaus AG zum Jahreswechsel geschickt hat: „Ist unsere Sprache noch zu retten?"

Zum Wort schneien gibt es im „Deutschen Wörterbuch" (herausgegeben und bearbeitet vom Wissenschaftlichen Rat und den Mitarbeitern der Dudenredaktion unter Leitung von Günther Drosdowski) 3 Einträge:

1.) unpersönlich (von Niederschlag), als Schnee zur Erde fallen, mit der neuen Übertragung n es schneit" (auf dem Bildschirm) für nes flimmert", Fernsehtechnik-Jargon,

2.) in grober Menge wie Schnee herabfallen und

3.) umgangssprachlich: unerwartet überraschend an einem bestimmten Ort, zu jemandem kommen.

Fehlt also beim Stichwort schneien noch die neue „ununpersönliche”, das heißt auch transitive Verwendung. Möglich wäre etwa der folgende Satz: Kleinkirchheim schneit hervorragendes Material, Simonhöhe schneit pappiges Zeug. Es verzeichnet das Wörterbuch aber beim Verbum beschneien bereits die neue Bedeutung: ${ }_{n}$ mit einer Schicht von künstlichem Schnee bedecken" und Beschneiung, "das Beschneien mit Kunstschnee”. Hier war der Weg vom Zustandspassiv die Landschaft ist beschneit zum Vorgangspassiv die Landschaft wurde beschneit auch sichtlich kürzer und "naheliegender" als vom unpersônlichen es schneit zum persönlichen Kleinkirchheim schneit.

Bad Kleinkirchheim schneit. In dieser Wendung schneien als „persönlich" verwendet anzusprechen, geht genau genommen nicht an. Denn eine Ortschaft ist keine Person. Ein Ortsname würde sich per definitionem gut für einen „Umstand des Ortes" ein „Lokaladverbiale”, auch für ein "präpositionales Attribut" eignen, ein „Subjekt aber ist eine Ortschaft nicht eigentlich oder im Sinne einer Gebietskörperschaft, einer Kommune, einer „juristischen Person". Ein solches Subjekt kann manches, aber vermag semantisch nicht alles. Man schneit in Bad Kleinkirchheim verbalisiert den neuen Sachverhalt genauer wie: Es schneit in Bad Kleinkirchheim den alten, nennen wir ihn den „mythischen Sachverhalt”. Bad Kleinkirchheim schneit leistet eine Sachverhaltsdarstellung somit nur rhetorisch und uneigentlich. Genaugenommen haben wir es mit der Fi- 
gur der Metonymie oder einer besonderen Figur der Metonymie zu tun, bei der das eigentliche Subjekt nicht proprie bezeichnet, sondern ein semantisch benachbartes und verwandtes Wort verwendet wird. Die Ortsbezeichnung statt des Bürgermeisters oder Fremdenverkehrsdirektors des Ortes, die das Schneien anordnen, oder die Gemeindebediensteten, die tatsächlich auf Anordnung des Vorgesetzten hin schneien, ist freilich ein naheliegender, rhetorisch gesprochen signifikanter Ersatz. Gerade auch im Vergleich der Ortschaften ist die Ortsbezeichnung (wie im Schneebericht oder im Wetterbericht) eine zentrale Information. Die entsprechenden Ortschaften sind auch ausgesprochene Wintersportorte, schon das sagt alles, die Ortschaftsnamen sind oft geradezu ${ }_{n}$ Synonyme" für eine herausragende Fremdenverkehrsveranstaltung, die an dem entsprechenden Ort stattfindet, sie sind abgestempelt und haben eine „Nenotation". Mit Attnang verbinden die Österreicher das Umsteigen von einem Zug in einen anderen (Bahnknotenpunkt). Und wenn ich eine andere $\mathrm{Me}$ tonymie höre, etwa Bonn dementiert oder Moskau wartet ab, so weiß ich auch ungefähr, was aus den betreffenden Städten als Capitalia zu erwarten ist und um was es sich wohl handeln muß. „Rom tritt auf die Bremse" las ich kürzlich, womit gemeint war, daß die Reformen des 2. Vatikanischen Konzils verlangsamt oder zurückgenommen werden.

In einem Artikel „Die unheimliche Siegesserie Girardellis” (Die Presse, 18. Jänner 1989) las ich, daß sich der Sieger der Hahnenkammabfahrt auf Wengen freut: „Am liebsten”, meint er, „am liebsten wär mir, sie würden alles vereisen. Dann wird's wenigstens keine Materialfrage". Sieht man nun ins „Deutsche Wörterbuch", so findet man bei vereisen zwei Einträge:

1.) sich durch gefrierende Nässe mit einer Eisschicht überziehen (seltener "sich mit Eis bedecken", zufrieren), und

2.) „einen Bereich der Haut durch Aufsprühen eines bestimmten Mittels für kleinere Eingriffe unempfindlich machen".

Der neue Gebrauch des Wortes vereisen kommt also im Wörterbuch noch nicht vor. Vereisen als ein systematisches Besprengen des Schnees der Piste, um ihm Halt, Festigkeit oder bestimmte Eigenschaften zu verleihen. Jenes Vereisen dient, wie man lesen kann, auch dazu, eine "selektive Strecke" herzustellen und eine spektakuläre Konkurrenz zu bekommen, mit Streckenrekorden und Höchstgeschwindigkeiten - und damit auch „spektakulären” Stürzen! Das Fremdwort selektiv bedeutet im sozialdarwinistischen Sinne „die Unterschiede zwischen guten und schlechten Läufern herausstellend und deutlich machend", so daß "nicht das Material, sondern das Können des Fahrers entscheidet".

Ich glaube nicht, daß es einen vernünftigen Grund gibt, warum man 
die Funktionserweiterung der alten "Schneewörter" ablehnen oder negativ bewerten sollte. Auch wenn man die systematische Ausbeutung von Natur und Landschaft für den Tourismus ablehnt und wenn man die Schäden, die durch Abholzen der Wälder für Trassen, Pisten und Liftschneisen, das Präparieren der Schneedecke mit Schneezement und so weiter bedauert, so wird man doch den Ausdruck all dessen in der Sprache nicht bedauern können, was bekanntlich einer Verwechslung von Landschaft und Landkarte gleichkäme. Denn auch dann, wenn man über den neuen materialistischen Zeitgeist und die totale Vermarktung der Natur unzufrieden ist, braucht man Wörter, um diese Unzufriedenheit artikulieren zu können, eine entsprechende Sprache. Wer sich etwa gegen die Valenz- oder Rektionsveränderung des Wortes schneien, die gerade stattfindet, sträuben möchte, der schlüge tatsächlich den Sack statt des Esels. Ja man kann in jenem Wandel vom unpersönlichen zum persönlichen Gebrauch geradezu eine "flächendeckende", "das zugrundeliegende Phänomen restlos beschreibende und einholende" Ausdrucksmöglichkeit erblicken - und begrüßen. Die Sprache bringt mit dem persönlichen Gebrauch des Wortes schneien die stattfindende technische Veränderung auf den Punkt. So wie mit Hilfe der Technik natürliche Grenzen übersprungen werden, und wie dem natürlichen Mangel durch technische Hilfsmittel und Surrogate (Kunstschnee statt natürlichem Schnee) abgeholfen wird, so wird die grammatische "Beschränktheit" überwunden. Mit der neuen technischen Aktion als Reaktion auf natürliche Defizite wird auch sprachlich die Aktionsart - und damit der Aktionsradius der Wörter und ihrer grammatisch-syntaktischen Möglichkeiten - erweitert. So wie die Strecke wird auch $d_{\mathrm{Ac}}$ jprache selektiv, es gibt also jenen Sprecher, der auf der Höhe der Zeit die neuen Sachverhalte angemessen präzise, prägnant, mit wenig Umschreibungen und paraphrastischer Schwerfälligkeit ausdrückt, ökonomisch und effizient verfährt und dabei neue Wege beschreitet, die Sprache nützt und benützt, um mit nützt und benützt auch den Zusammenhang von System und Äußerung (Kompetenz und Performanz) anzusprechen. Und es gibt den langsamen und schwerfälligen Sprecher, der zum Fallen und Stürzen neigt. Er ist ein Opfer der selektiv gewordenen Sprache.

Wie im Falle des schneien, das nun die Schneetechniker und die Fremdenverkehrsdirektoren "in die Hand genommen haben", wovon die Grammatiken und älteren Wörterbücher, ja auch nicht nur die älteren Wörterbücher nichts wissen, wurde auch das Wort rechnen um eine Anwendungsmöglichkeit bereichert und um den unpersönlichen rückbezüglichen Gebrauch erweitert: Es oder etwas rechnet sich, etwas muß sich rechnen.

Reich ist etwa wieder der Artikel im „Deutschen Wörterbuch” zum 
Lemma rechnen, fünfgeteilt mit a- und b-Unterabteilungen, jener Gebrauch aber, der impersonale reflexive, der prägnant die Notwendigkeit schwarzer Zahlen, des Vermeidens der roten Zahlen ausdrückt, im Sinne von "es muß sich auszahlen, es muß sich rentieren", „es muß unter dem Strich ein Plus herauskommen", „es muß ökonomisch sein", findet sich nicht darunter. Dabei scheint es, daß im Reflexivum das alte Medium, die mittlere Aussageart zwischen Aktiv und Passiv Urstände feiert.

Ein anderes reflexiv gewordenes oder doch in neuer rückbezogener idiomatischer Weise verwendetes Wort ist mir neulich beim Radiohören aufgestoßen, eine Redewendung der Jugendsprache wohl - und sie wird hier in Mannheim längst nicht mehr unbekannt, sondern bereits verbucht sein: Es wurde also im Österreichischen Rundfunk (Ö3) verlautbart, daß derjenige, „der sich den Davis-Cup im Dusika-Stadion geben möchte", bei einem folgenden Quiz für die an sich ausverkaufte Veranstaltung noch Karten gewinnen könne. Sich ein Popkonzert geben, aber auch negativ verwendet: Heute geb ich mir die Vorlesung nicht ("lasse die Vorlesung ausfallen", von den Hörern, nicht dem Dozenten, gesagt), sei unter Jugendlichen sprachlich allgemein der Brauch. Von Beziehungen einer Lehnübersetzung etwa aus dem Englischen abgesehen, erscheint dieses neue sich etwas geben eine Nähe zu medizinischer Ausdrucksweise zu haben: etwas eingeben, etwas einnehmen, man denke auch an die Gabe im medizinischen und chemischen Jargon. Von der Medizin aber ist nur ein kleiner Schritt zur Nomenklatur der Drogenszene er gibt sich die Spritze, den Goldenen Schuß, wie früher: die Kugel, und sicher konnotieren im semantischen Umfeld der neuen Redensart auch die Wendungen das gibt mir etwas, das gibt mir Berge, das gibt mir nichts. Und man muß kein extremer Zeitgeistkritiker oder Kulturpessimist sein, wenn man in ich gebe mir ein universales, semantisch unspezifisches, aber multifunktionales Syntagma des Konsums, der Instrumentalisierung der Person oder auch des Leibes erblickt. Der Geist oder Verstand wird als eine körperunabhängige oder personfremde Instanz fingiert, die dem Körper oder der Person etwas zuteilt, verordnet, genehmigt oder zukommen läßt - oder das Gegenteil. (Man kann darin auch eine Parodie des "herrschaftlichen Diskurses" sehen). Und von hier ist nur ein kleiner ideologischer Schritt zum sich rechnen, denn das, was man sich gibt, muß sich - im direkten oder doch übertragenen Sinn - rechnen. Und auch der Kreis zu „Ski total", eines jener Jahreszeitvergnügen, ist schon geschlossen: ich gebe mir Skifahren, wenn es sich rechnet.

Das Produkt, das die Schneekanonen erzeugen, heißt ${ }_{n}$ Kunstschnee", künstlicher Schnee, oder noch häufiger erscheint in den Berichten über Wintersportorte der Ausdruck „Kompaktschnee”. Es lohnt sich, die se- 
mantische Tiefe dieses Wortes anzusehen. Kompaktschnee verzichtet oder umgeht den Ausdruck künstlich, die Position „künstlich" erscheint somit im Sinne des Differentials plus künstlich und minus künstlich, Kompaktschnee könnte also, muß aber nicht künstlich sein. Denkbar ist dem Worte nach „natürlich oder künstlich geschneiter Schnee". Kompaktschnee vermeidet also das Ansprechen der Künstlichkeit, des Surrogathaften, denn Skivergnügen soll im Marketing als Naturvergnügen verkauft werden, nicht als Verlegenheit. Wer es sich leisten kann, leistet sich echtes Leder nicht Lederersatz. Kompakt hat den Vorteil, ein Fremdwort zu sein, und zwar ein angesehenes. Kompakt ist das Gegenteil von diffus. Was "kompakt" ist, braucht wenig Raum, ist also ökonomisch, - hier stellt sich die Assoziation zur „Compactdisc" ein, die es zum allgegenwärtigen Kürzel $C D$ gebracht hat. Kompaktschnee wäre demnach CS (Si Es). Das Wort ist auf dieser assoziativen Basis durchaus werbefähig und werbewirksam, ohne die Problematik des „Kompakten" in der Zusammensetzung Kompaktschnee ganz zu verschweigen. Broder Castensen kennt in seinem 1965 erschienenen Buch "Englische Einflüsse auf die deutsche Sprache nach 1945" natürlich nicht die "Compactdisc", erwähnt aber den "Kompaktwagen": compact car (NSU Prinz, S. 239). Hier wird freilich auch der Mangel der Kleinheit euphemistisch nominiert, wenn das Kompakte natürlich auch echte Vorteile bedeutet. Denn wenn die "Komprimiertheit" oder "Kompaktheit" in vielen Fällen ein Positivum ist, - wie bei den kleinen, raumsparenden Schallplatten -, so muB sie dies nicht auch in allen anderen sein. In Kompaktschnee deutet, recht verstanden, das kompakt auf eine sehr fragwürdige Eigenschaft des Kunstschnees hin, denn kompakt ist hier eigentlich ein Synonym für pappig, matschig, wässrig; kompakt ist somit das Gegenteil von pulvrig im Sinne des Pulverschnees, auch das Gegenteil von gführig ${ }_{n} Z$ wa Brettln a gführiger Schnee juchee"). Und das Fahren auf Kompaktschnee ist sicherlich auch kein "Tiefschneefahren abseits der Pisten", für das sonst auch Werbung gemacht wird. So hört sich das Wort Kompaktschnee zwar gut an, kann aber auch als Warnung verstanden und gelesen werden, es macht das Beste aus der schlechten Situation, klingt gut oder nicht schlecht, ohne expressis verbis zu lügen, kompakt nennt eine in den übrigen Fällen von Komposition positiv notierende Eigenschaft, die freilich in diesem Fall ins Negative umschlägt. Haare aber sind schön am Haupt, nicht in der Suppe. Kompaktschnee ist also sicherlich keine einklag- oder einforderbare Irreführung, keine leere Versprechung. Wer es miBversteht, ist selbst schuld.

Der Sprachkritiker herkömmlicher Prägung hätte also, wenn er sich allein das Wortfeld „Wintersport” heute ansieht, eine Menge Stoff für kritische Anmerkungen und gute oder weniger gute Gründe zum Ärgern. 
Wenn er etwa davon ausgeht, daß es bei den alten „Schneewörtern" bleiben müsse oder auch bei der alten Verwendung, auch in semantischer und syntaktischer Hinsicht, dann fände er sicher Anlaß zur Schelte. Gerade auch auf dem beliebtesten Gebiet der Sprachkritik, dem Monieren der Fremdwörterei, findet sich Stoff und Munition für die Kritik. Gar nicht nur unbegründete Kritik. Denn natürlich ist es nicht einzusehen, daß das Deutsche keine eigenen Ausdrücke für verschiedene neue Sportgeräte oder sportliche Betätigungen entwickelt, sondern sich nur noch von den Amerikanern bedienen läßt. Man könnte mit einigem Recht sagen, hier handle es sich um einen alpinen Sport oder um einen nordischen Sport und so sollte das Deutsche als eine der alpinen Sprachen auf diesem Gebiet weiter eine Rolle spielen. Freilich käme man bei dieser Argumentation insofern in Schwierigkeiten, als die Kernwörter des Wortfeldes, etwa $S k i$ oder Slalom oder Telemark nicht deutsch sind - und so germanisch wie $S k i$ ist $S$ nowboard noch allemal. Trotzdem darf einem durchaus zu denken geben, daß die großen französischen, Schweizer, österreichischen und jugoslawischen und "deutschen" Wintersportgerätehersteller ihre Produkte „Dynamic”, „Atomic”, „Quattro”, "Blizzard Vacuum" nennen. Sie geben damit einfach zu erkennen, wo sie sich das große Geschäft erwarten, so wie eben die Wirtschaftswelt angloamerikanisch ausgerichtet ist. Sie zeigen „vorauseilenden Gehorsam" im Sprachlichen, unterwerfen sich Zwängen. ( $40 \%$ der sogenannten Fremdwörter sind hausgemacht!) Sie sind entschuldigt. Heroen aber sind sie nicht. Um im Geschäft mitzumischen, müssen sich die Japaner sicher noch weit mehr verleugnen als die Tiroler Skifabrikanten. Die Tiroler sprechen auch den Japanern zuliebe Englisch... Wenn man etwa die japanischen Autonamen ansieht, so handelt es sich um stromlinienförmige Nipponismen, die ihre Herkunft aus gutem Haus ansprechen, aber den "fremdsprachigen" Markt auch nicht überfordern, es sind im Amerikanischen integrierbare Appelativa. Bedenklich wird es sicher aber für eine nichtamerikanische Sprache dort, wo alte Erbwörter verdrängt werden und amerikanische Modewörter auftauchen. Es kann einem zum Beispiel kaum jemand erklären, warum und zu welchem Ende das Deutsche das Wort Jogging importiert hat, wo das Wortfeld "Laufen" im Deutschen eigentlich nicht gerade arm bestückt ist. Denn "Dauerlauf" wurde doch eigentlich schon vor dem „Jogging” praktiziert, der "Jogginganzug” aber ist allenfalls ein modifizierter "Trainingsanzug", ohne daß ich behaupten möchte, daß es sich beim alten Wort Training um ein Wort des deutschen Kernwortschatzes handelt. Auch vor dem Ausdruck Shopping wurde in unseren Städten schon "eingekauft" oder "zum Einkaufen gegangen", gab es den „Einkaufsbummel”, wenn auch mit Shopping möglicherweise auf die Ausdauer, die Lust an jener Tätigkeit über die vitalen Interessen 
hinaus und eine neue Ritualität des Gustierens hingedeutet werden mag.

Im Wortfeld "Wintersport" ist vor einigen Jahren eine neue Disziplin und ein neues, das heißt fremdes Wort für diese Disziplin aufgetaucht. Ich spreche aus der Sicht des Deutschsprachigen. Sachlich handelte es sich neben Slalom, Torlauf und Abfahrt um einen vierten Bewerb, der ein Zwischending $\mathrm{zwischen}$ Torlauf und $\mathrm{Abfahrt}$ ist, einen Bewerb, restriktiver als die Abfahrt, aber liberaler als der Torlauf. Die FIS (ein englisch-französisches Initialwort für den Internationalen Schiverband) gab dem neuen Wettbewerb den Namen "Super G" für "Super Giant Slalom". Auch französisch läßt sich das Kürzel Super $G$ auflösen $(S u$ per Gêant). Für viele Österreicher aber ist das $G$ (dschi) schwer- bis unverständlich. Und wenn auch die Sportreporter wissen werden, was die Abbreviatur dechiffriert und aufgelöst bedeutet, so ist diese Kenntnis beim Großteil der Zuseher von Schiübertragungen nicht gegeben. Es hat mittlerweile auch eine volksetymologische Umdeutung des Dschi (G) auf Schi stattgefunden, so daß viele statt "Dschi" "Schi" sagen und damit zu erkennen geben, welche Erklärung sie für das fremde Kürzel gefunden haben. Die erwähnte Umdeutung wird auch dadurch gestützt und ermöglicht, daß auch in der Aussprache der Kompetenten das anlautende $d$ in $d s c h i$ im Ergebnis einer Art „Metanalyse”, also einer fehlerhaften Herauslösung eines Wortes aus einer Verbindung, an das vorhergehende Wort Super angehängt wird, so daß Superd Schi entsteht. Durch die sprachübliche und richtige Betonung des ersten Wortes Super im Sinne einer "differentia specifica" gerät das $G$ auch in den Tonschatten, wodurch es zusätzlich für Formveränderung und Substanzverlust, also Gestaltungsschwund, anfällig wird. Auf diesen Verstehensund Mißverständnis-Wegen ist der "Super G" im österreichischen sprachlichen Alltag nun bei „Super(d)Schi” gelandet, als hätte sich der SchiNormalverbraucher nun durchgesetzt und die fremde Sache auf einen einheimischen Nenner gebracht.

Günther Drosdowski hat in seinem Vortrag "Ist unsere Sprache noch zu retten" auf besonders drastische und affige Beispiele von "Anglophilie" etwa im Bereich der Medien und des Rundfunks hingewiesen. Bei "Jogging" oder "Shopping" etwa handelt es sich doch nicht eigentlich um eine Neuentdeckung oder absolute Innovation, allenfalls eine Wiederentdeckung und „Renovation", die Betonung oder Neubewertung einer aus der Mode gekommenen Betätigung, eine Rechtfertigung für Verbalimperialismus und Sprachkolonialismus kann in dieser "Leistung" nicht gesehen werden, wenn man davon ausgeht, daß die Erfindung oder Erzeugung eines Produkts dem Erfinder oder Erzeuger das Recht gibt, sich auch sprachlich im Rahmen der Nomenklatur zu behaupten und durch- 
zusetzen. Den Deutschen und dem Deutschen wurden aber in den letzten Jahren nicht wenige Ladenhüter angedreht. Die nationalsprachliche Welt stürat deshalb sicher nicht zusammen.

Ich habe mir bei meiner Arbeit an einer Reihe von sprachkritischen Glossen für das "Spektrum" der Wiener Tageszeitung "Die Presse", die ich demnächst mit der Nummer 40 beende, immer die von Wolfgang Klein in der Nummer 62 der „Zeitschrift für Literaturvissenschaft und Linguistik" formulierten Bewertungsgrundsätze für sprachliche Phänomene, vor allem für Veränderungen, vor Augen gehalten, die er als "Angemessenheit", „Verständlichkeit", „Wohlgefallen" und "Einfachheit" formuliert hat. Bei den Veränderungen, unter die auch der Wortimport fallen würde, sehe ich gern auf ein Kriterium, das ich als "Notwendigkeit" bezeichnen würde. Für viele Veränderungen scheint diese Notwendigkeit nicht gegeben, so daß man zwar den Grund erkennt, aber keinen Sinn in der Veränderung erblicken kann. Es ist durchaus verständlich, daß sich jemand mit Fremdwörtern oder fremdsprachlichen Phrasen ein Ansehen geben möchte, wie ja das Imponieren ein menschlicher Impuls ist, (so wie eine ansprechende Kleidung), es ist aber auch verständlich, daß der Sprachkritiker die Wörter semantisch analysiert und wenn er in einem bloßen Snobismus oder verbalen Modernismus den einzigen Grund für ihre Verwendung erkennt, urteilend und verurteilend auf den Plan tritt, also auch im Rahmen seiner sprachpädagogischen Möglichkeiten „sprachlenkerisch" tätig wird - eingedenk der grundsätzlichen Fragen, ob Sprachlenkung möglich, notwendig und wünschenswert ist.

So erfüllen, wie ich an einigen neuen Verwendungen von "Nivea", also "Schneewörtern", oder Wörtern des Wortfeldes "Winter" zu zeigen versuchte, die Forderung 1 Wolfgang Kleins:

Verantwortung gegenüber der Sache: Das, was ausgedrückt werden soll, muß so ausgedrückt werden (können), daß in der Tat ausgedrückt wird, was ausgedrückt werden soll.

Das „Schneien"-Beispiel hat gezeigt, wie die neue Ausdrucksweise der neuen Situation angepaßt erscheint. Und da mit dem personalen Gebrauch des Wortes auch die anderen 3 Kriterien erfüllt erscheinen oder doch nicht gröblich verletzt

Verantwortung gegenüber dem Hörer bzw. Leser: Eine Sache muß so ausgedrückt werden (können), daß sie der jeweils angesprochene Leser Leser bzw. Hörer verstehen kann, und zwar möglichst gut und leicht;

Verantwortung gegenüber den Musen: Was gesagt wird, soll nicht nur die Sache, die ausgedrückt wird, genau wiedergeben und für den jeweils Angesprochenen verständlich sein, es soll auch schön, elegant, witzig 
ausgedrückt sein, es soll gefallen;

Einfachheit: Das, was ausgedrückt wird, soll möglichst einfach ausgedrückt werden (können); insbesondere soll es möglich sein, nur das auszudrücken, was nach den ersten beiden Kriterien erforderlich ist,

- da somit kein vernünftiger Grund gegen die Änderung spricht, wenn man sich nicht auf den rigorosen Standpunkt stellt, es müsse alles ein für allemal beim alten bleiben, was eine intellektuelle Albernheit wäre, kann man diese Veränderung - nur als Beispiel - gerne gutheißen. Auf das Thema zurückformuliert, nicht jede Veränderung ist ein Qualitätsverlust, aufs Ganze gesehen ist nicht jede Veränderung, aber die Veränderungsfähigkeit, ein Garant für Adaption - und Qualitätskonstanz. Und wenn man sich auch vor allen biologischen Vergleichen hüten soll, weil gerade im Biologismus eine der Wurzeln für den Mythos (oder Wahn) vom Sprachverfall gesehen wird (vgl. W. Klein), so kann man die veränderte Sprache mit der erweiterten und veränderten Medizin vergleichen, mit der man den alten und den neuen Krankheiten zu Leibe rückt. Mit Aderlassen oder Blutegelansetzen kommt man nicht mehr zu Rande. Die Sprache wird nicht schlechter oder besser. Sagen wir, der Sprachgebrauch (denn die Verwendung ist das, was der Sprachkritiker in den Blick nimmt), die Sprachverwendung ist zu allen Zeiten relativ gut, das heißt aber auch relativ schlecht, also kritikwürdig. 